\title{
A micropapillary pattern is predictive of a poor prognosis in lung adenocarcinoma, and reduced surfactant apoprotein A expression in the micropapillary pattern is an excellent indicator of a poor prognosis
}

\author{
Hideaki Tsutsumida ${ }^{1}$, Mitsuharu Nomoto ${ }^{1}$, Masamichi Goto ${ }^{1}$, Shinichi Kitajima ${ }^{1}$, \\ Ichiro Kubota ${ }^{2}$, Yasunobu Hirotsu², Joeji Wakimoto ${ }^{3}$, Michael A Hollingsworth ${ }^{4}$ \\ and Suguru Yonezawa ${ }^{1}$
}

${ }^{1}$ Department of Human Pathology, Field of Oncology, Kagoshima University Graduate School of Medical and Dental Sciences, Kagoshima, Japan; ${ }^{2}$ Department of Surgery, National Minamikyushu Hospital, Kagoshima, Japan; ${ }^{3}$ Department of Pathology, National Minamikyushu Hospital, Kagoshima, Japan and ${ }^{4}$ Departments of Biochemistry and Molecular Biology, Eppley Institute for Research in Cancer and Allied Diseases, University of Nebraska Medical Center, Omaha, NE, USA

\begin{abstract}
A micropapillary pattern is defined as papillary tufts without a fibrovascular core and is known to be a factor that indicates a poor prognosis in numerous cancers. However, their role in lung adenocarcinoma has not been investigated widely. In 185 cases of small-size lung adenocarcinoma $(\leq \mathbf{3} \mathrm{cm})$, cases with a micropapillary pattern ratio of more than $1 \%$ (analyzed by NIH image) were defined as micropapillary pattern positive. Correlations between the micropapillary pattern and clinicopathological factors were investigated and immunohistochemical expression of mucin and various antigens was examined in regions with and without micropapillary patterns. Micropapillary pattern-positive tumors (micropapillary pattern ratio $\geq 1 \%$ ) were observed in $11.4 \%$ of cases $(21 / 185)$ and the micropapillary pattern ratio correlated with TNM stage $(P=0.0002)$, lymphatic invasion $(P=0.0002)$ and lymph node metastasis $(P=0.03)$. Disease-free interval $(P<0.0002)$ and survival $(P=0.027)$ were significantly shorter for micropapillary pattern-positive patients, and micropapillary pattern-positive stage IA cases also had a significantly shorter disease-free interval $(P<0.0001)$. MUC1 was expressed strongly across the surface of the micropapillary structure, whereas MUC4 tended to show lower expression in the micropapillary pattern. It was noteworthy that the disease-free interval in patients with high surfactant apoprotein A expression was significantly better than in patients with low surfactant apoprotein $A$ expression $(P=0.03)$, and no recurrence or death occurred in patients with high surfactant apoprotein A expression. Our results show that the micropapillary pattern ratio correlates with lymphatic invasion and lymph node metastasis, and that a high micropapillary pattern ratio leads to a poor prognosis. High MUC1 expression on the surface is an important characteristic of a micropapillary pattern, and reduced surfactant apoprotein $A$ expression in the micropapillary pattern may be an excellent indicator for poor prognosis in small-size lung adenocarcinoma.
\end{abstract}

Modern Pathology (2007) 20, 638-647; doi:10.1038/modpathol.3800780; published online 13 April 2007

Keywords: lung cancer; micropapillary pattern; prognosis; MUC1; surfactant apoprotein A

Correspondence: Dr M Nomoto, MD, Department of Human Pathology, Field of Oncology, Kagoshima University Graduate School of Medical and Dental Sciences, 8-35-1 Sakuragaoka, Kagoshima 890-8544, Japan.

E-mail: sanchan@m2.kufm.kagoshima-u.ac.jp

Received 7 November 2006; revised 15 February 2007; accepted 16 February 2007; published online 13 April 2007
Lung cancer is a disease with a poor prognosis, despite efforts in primary prevention, screening and therapy. However, many small lung adenocarcinomas without symptoms can now be detected due to advances in diagnostic techniques. Such adenocarcinomas are becoming an important area of study, but many show metastasis or recurrence. To improve the cure rate of small-size lung adenocarcinoma, it is important to make a clear histopathological 
distinction between carcinomas of patients with favorable outcomes and those with poor outcomes.

A micropapillary pattern is defined as a pattern showing micropapillary structures without a fibrovascular core. It is well known that the presence of a micropapillary pattern leads to a poorer prognosis in ovary, breast and bladder cancer. A micropapillary pattern has also been reported as an important factor in predicting poor prognosis in lung adenocarcinoma, ${ }^{1-6}$ but the immunohistochemical profile of micropapillary patterns in lung adenocarcinoma is not well understood.

In this study, we determined the micropapillary pattern ratio in small-size lung adenocarcinoma samples using a more objective method than used previously, and we examined the relationship between the presence of a micropapillary pattern and prognosis. We suggest that the unique char- acteristics of a micropapillary pattern lead to a poorer prognosis in cases with a high micropapillary pattern ratio.

\section{Materials and methods}

\section{Surgical Specimens and Patient Characteristics}

From January 1994 to December 2000 in the Minami Kyushu National Hospital, radical surgical removal was performed for 185 patients (males, 72; females, 113; mean age, 67.0 years old (range 43-84)) with primary lung adenocarcinoma of the non-BAC type and smaller than $3 \mathrm{~cm}$ in size. Histologically, the adenocarcinoma was grade 1, 2 and 3 in 106, 76 and three cases, respectively. The patient characteristics are shown in Table 1. Distant metastasis was not detected in any patient and performance status was

Table 1 Correlation between clinicopathologic factors and presence of a micropapillary pattern in small-size lung adenocarcinoma

\begin{tabular}{|c|c|c|c|c|c|c|}
\hline \multirow[t]{2}{*}{ Category } & \multirow[t]{2}{*}{ No. of patients } & \multicolumn{2}{|c|}{ Log-rank test (P-value) } & \multicolumn{2}{|c|}{ Micropapillary pattern } & \multirow[t]{2}{*}{$\mathrm{P}$-value } \\
\hline & & Disease-free interval & Overall survival & Negative $(\mathrm{n}=164)$ & Positive $(\mathrm{n}=21)$ & \\
\hline \multicolumn{7}{|l|}{ Age (years) } \\
\hline$\leq 65$ & 81 & 0.26 & 0.11 & $68(36.8)$ & $13(7.0)$ & 0.076 \\
\hline$>65$ & 104 & & & $96(51.9)$ & $8(4.3)$ & \\
\hline \multicolumn{7}{|l|}{$\operatorname{Sex}$} \\
\hline Male & 72 & 0.012 & 0.1 & $63(34.1)$ & $9(4.9)$ & 0.69 \\
\hline Female & 113 & & & $101(54.6)$ & $12(6.5)$ & \\
\hline \multicolumn{7}{|l|}{ TNM stage } \\
\hline IA & 131 & $<0.0001$ & 0.0002 & $124(67.0)$ & $7(3.8)$ & 0.0002 \\
\hline IB & 7 & & & $7(3.7)$ & $0(0)$ & \\
\hline IIA & 15 & & & $10(5.4)$ & $5(2.7)$ & \\
\hline IIB & 2 & & & $1(0.54)$ & $1(0.5)$ & \\
\hline IIIA & 25 & & & $19(10.3)$ & $6(3.2)$ & \\
\hline IIIB & 5 & & & $3(1.6)$ & $2(1.1)$ & \\
\hline \multicolumn{7}{|l|}{$P$-factor } \\
\hline P0 & 145 & $<0.0001$ & 0.0002 & $130(70.1)$ & $15(8.1)$ & 0.34 \\
\hline $\mathrm{P} 1$ & 28 & & & $24(13.0)$ & $4(2.2)$ & \\
\hline $\mathrm{P} 2$ & 10 & & & $9(4.9)$ & $1(0.5)$ & \\
\hline $\mathrm{P} 3$ & 2 & & & $1(0.5)$ & $1(0.5)$ & \\
\hline \multicolumn{7}{|l|}{ Tumor size } \\
\hline$<20 \mathrm{~mm}$ & 71 & 0.001 & 0.0065 & $66(35.7)$ & $5(2.7)$ & 0.144 \\
\hline$\geq 20 \mathrm{~mm}$ & 114 & & & $98(53.0)$ & $16(8.7)$ & \\
\hline \multicolumn{7}{|c|}{ Lymph node metastasis } \\
\hline$<\mathrm{N} 1$ & 159 & $<0.0001$ & $<0.0001$ & $144(78.3)$ & $15(8.2)$ & 0.033 \\
\hline$\geq \mathrm{N} 2$ & 25 & & & $19(10.3)$ & $6(3.3)$ & \\
\hline \multicolumn{7}{|c|}{ Lymphatic invasion } \\
\hline Negative & 117 & $<0.0001$ & $<0.0001$ & $111(61.3)$ & $6(3.3)$ & 0.0002 \\
\hline Positive & 64 & & & $49(27.1)$ & $15(8.3)$ & \\
\hline \multicolumn{7}{|c|}{ Venous invasion } \\
\hline Negative & 142 & $<0.0001$ & $<0.0001$ & $127(69.8)$ & $15(8.2)$ & 0.438 \\
\hline Positive & 40 & & & $34(18.9)$ & $6(3.3)$ & \\
\hline \multicolumn{7}{|l|}{ Serum CEA } \\
\hline Negative & 136 & $<0.0001$ & 0.0012 & $122(69.3)$ & $16(9.1)$ & 0.85 \\
\hline Positive & 41 & & & 34 (19.3) & $4(2.3)$ & \\
\hline
\end{tabular}


0 or 1 in all patients. All specimens were fixed in $10 \%$ formalin, embedded in paraffin and cut into $4-\mu \mathrm{m}$ serial sections for immunohistochemical staining, in addition to the usual hematoxylin and eosin staining. This study was approved by the Kagoshima University Faculty of Medicine Human Investigation Committee (registration no. H13-19).

\section{Micropapillary Pattern Ratio}

Detection of micropapillary patterns was performed using the specimen of largest area for each sample. CD68 expression was analyzed immunohistochemically to exclude alveolar macrophages. The micropapillary pattern area was marked on the scanned specimen and the micropapillary pattern ratio (micropapillary pattern area/non-micropapillary pattern area $\times 100$ ) was analyzed using NIH image (version 1.62, NIH Division of Computer Research and Technology, Bethesda, MD, USA). Cases showing a micropapillary pattern ratio of $\geq 1 \%$ were defined as micropapillary pattern positive.

\section{Comparison of Micropapillary Pattern Ratio with Clinicopathological Factors}

Various potential prognostic parameters (age, sex, TNM stage, $P$-factor, tumor size, lymph node metastasis, lymphatic invasion, venous invasion and serum CEA level at the preoperative stage) were examined to determine their relationship with disease-free interval, overall survival and micropapillary pattern status (Table 1 ). The $P$-factor reflecting the degree of visceral pleural invasion was defined according to the criteria of the Japan Lung Cancer Society as follows: P0, the tumor does not penetrate the elastic layer of the visceral pleura; P1, the tumor penetrates the elastic layer but is not exposed on the pleural surface; P2, the tumor is exposed on the pleural surface but does not involve adjacent anatomic structures and P3, the tumor involves adjacent anatomic structures.

\section{Immunohistochemical Staining}

MUC1, MUC4, MUC5AC, surfactant apoprotein A, ErbB2, CEA, p16, p27 and CD68 expression was assessed by immunohistochemistry with the following antibodies: DF3 (mouse monoclonal antibody, Toray-Fuji Bionics, Tokyo, Japan) against MUC1 mucin; clone 8G7 (mouse monoclonal antibody) (generated by Surinder K Batra) against MUC4; clone CLH2 (mouse monoclonal antibody, Novocastra, Newcastle, UK) against MUC5AC; PE-10 (mouse monoclonal antibody, Dako A/S, Glostrup, Denmark) against surfactant apoprotein A; ErbB2 (rabbit polyclonal antibody, Dako A/S) against ErbB2; CEA (rabbit polyclonal antibody, Dako A/S) against CEA; clone G175-405 (mouse monoclonal antibody, BD
Bioscience Pharmingen, San Jose, CA, USA) against p16; NCL-p27 (mouse monoclonal antibody, Novocastra) against p27 and clone PG-M1 (mouse monoclonal antibody, Dako A/S) against CD68.

Immunohistochemical staining was performed with the immunoperoxidase method using the ABC complex (Vector Laboratories, Inc., Burlingame, CA, USA) as described previously. ${ }^{7,8}$ Briefly, each section was deparaffinized with xylene and endogenous peroxidase was blocked by incubating the sections in $0.3 \%$ hydrogen peroxide in absolute methanol at room temperature for $30 \mathrm{~min}$. After hydration in decreasing concentrations of ethanol in water, the sections were washed in $0.01 \mathrm{~mol} / \mathrm{l} \mathrm{PBS}$ (pH 7.4). Water bath pretreatment at $80^{\circ} \mathrm{C}$ for $20 \mathrm{~min}$ in $0.01 \mathrm{M}$ citrate buffer ( $\mathrm{pH}$ 6.0) was used for MUC4 epitope retrieval and water bath pretreatment at $98^{\circ} \mathrm{C}$ for $40 \mathrm{~min}$ was performed for ErbB2 epitope retrieval. Autoclave pretreatment at $120^{\circ} \mathrm{C}$ for $5 \mathrm{~min}$ was used for p27 epitope retrieval. All sections were washed twice with PBS and then exposed to $2 \%$ horse serum in PBS for $30 \mathrm{~min}$ at room temperature to prevent nonspecific staining. For staining using each antibody, the sections were incubated with primary antibodies (dilutions: DF3, 1:10; PE-10, 1:100; MUC4, 1:3000; ERbB2, 1:100; p27, 1:100; CEA, 1:500; MUC5AC, 1:100; p16, 1:250; CD68, 1:50) in PBS with 1\% bovine serum albumin for $16 \mathrm{~h}$ at $4{ }^{\circ} \mathrm{C}$ The sections were washed three times with PBS, incubated with the biotinylated secondary antibody and then washed three more times with PBS. All sections were then treated with the ABC complex (Vector Laboratories, Burlingame, CA, USA) for $30 \mathrm{~min}$. After washing a further three times with PBS, the sections were reacted with diaminobenzidine substrate for $10-30$ min for visualization, rinsed with tap water, counterstained with hematoxylin and mounted.

\section{Scoring of the Results}

Antibody staining was graded based on the percentage of positively stained neoplastic cells as follows: 0 , no neoplastic cells stained; $1+,<25 \%$ of neoplastic cells stained; $2+, \geq 25 \%$ to $<50 \%$ of neoplastic cells stained; $3+, \geq 50 \%$ to $<75 \%$ of neoplastic cells stained and $4+, \geq 75 \%$ of neoplastic cells stained. Staining of the cytoplasm, cell apex (luminal surface) and associated secretory products (luminal contents) was evaluated separately and cells were considered to be positive when at least one of these components stained positively. The immunohistochemical evaluation was performed by three observers ( $\mathrm{H}$ Tsutsumida, $\mathrm{M}$ Nomoto and S Yonezawa).

\section{Statistical Analysis}

The correlation of clinicopathological factors (age, gender, $T$-factor, tumor size, lymph node metastasis, 
lymphatic invasion, vessel invasion, serum CEA at the preoperative stage and smoking) with micropapillary pattern ratio was examined using univariate analysis. Statistical significance was set at $P<0.05$. Overall survival was measured from the date of surgery to the date of the last follow-up or death, whereas disease-free interval was measured from the date of surgery to the date when recurrence was first detected. Disease-free interval and overall survival curves were plotted using the KaplanMeier method and differences were analyzed by logrank test. Disease-free interval and overall survival were also compared between micropapillary pattern-positive (micropapillary pattern ratio $\geq 1 \%$ ) and micropapillary pattern-negative (micropapillary pattern ratio $<1 \%$ ) cases. JMP software (AS Institute Inc., Cary, NC, USA) was used for all statistical analyses.

\section{Results}

\section{Detection of a Micropapillary Pattern in Small-Size Lung Adenocarcinoma}

Micropapillary patterns were detected based on the presence of micropapillary structures without a fibrovascular core (Figure 1a). Expression of CD68 was analyzed to distinguish alveolar macrophages from a micropapillary pattern; alveolar macrophages show high expression of CD68 (Figure 1b) but low or no expression of MUC1 (Figure 1c). Among the 185 cases of adenocarcinoma, 53 included a micropapillary pattern. The micropapillary ratio ranged from 0.01 to $31.3 \%$ (mean \pm s.d.: $3.54 \pm 6.61 \%$ ) and the median value was $0.7 \%$. Six cases had a micropapillary pattern ratio of over $5 \%, 15$ had a micropapillary pattern ratio of $1-5 \%, 32$ had a micropapillary pattern ratio of $0-1 \%$, and 132 cases showed no
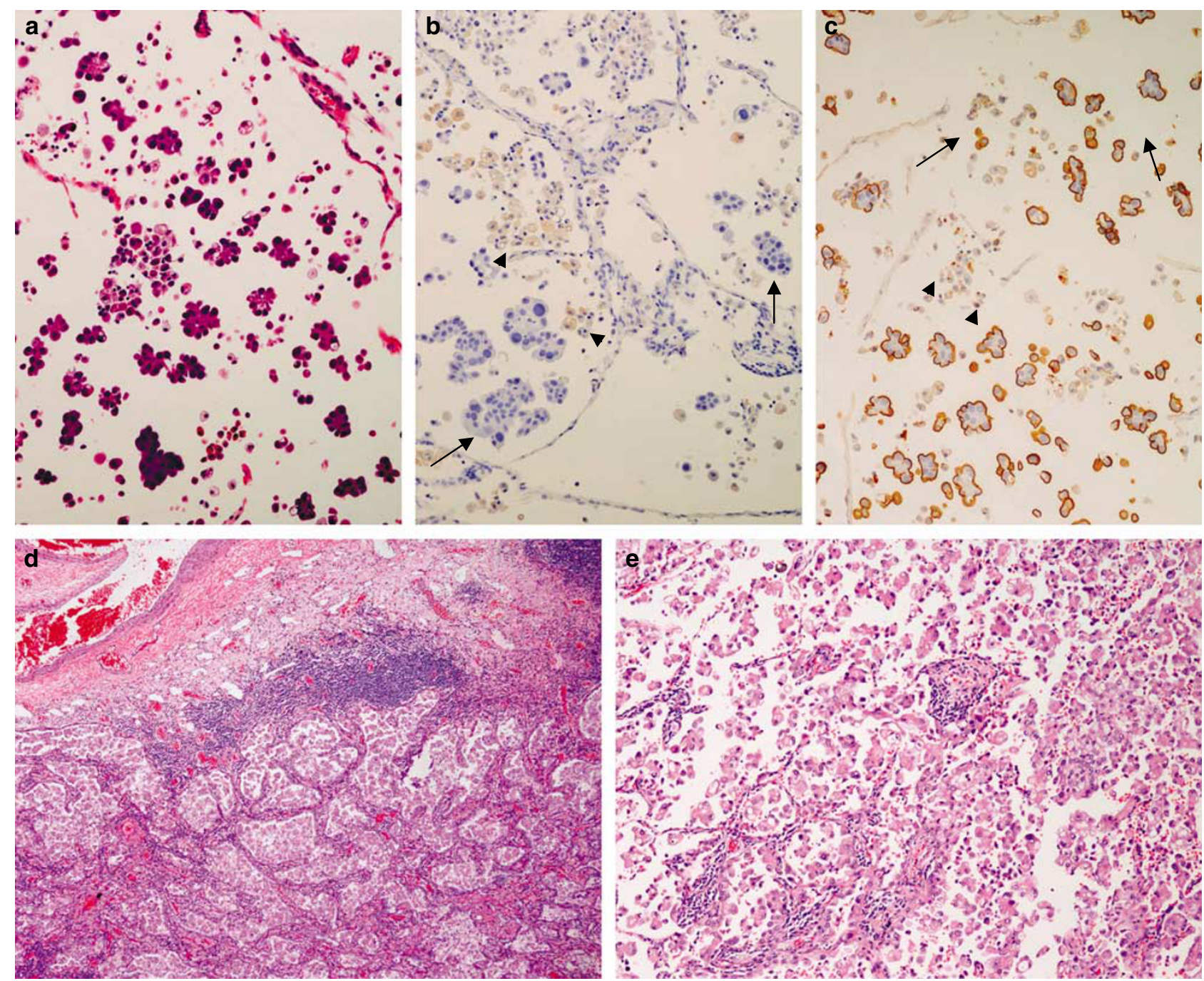

Figure 1 Typical H\&E and immunohistochemical findings in micropapillary pattern-positive cases. (a) H\&E staining. (b) Expression of CD68 was observed in macrophages (arrow head) but not in carcinoma cells with a micropapillary pattern (arrow). (c) Expression of MUC1 was observed in carcinoma cells with a micropapillary pattern (arrow) but not in macrophages (arrow head). (d) A micropapillary pattern in the space entrapped by atypical cells (inside pattern). (e) A micropapillary pattern in the normal alveolar space (outside pattern). 
Table 2 Micropapillary pattern ratio and definition of micropapillary pattern-positive and micropapillary pattern-negative cases

\begin{tabular}{lrc}
\hline $\begin{array}{l}\text { Micropapillary } \\
\text { pattern ratio }\end{array}$ & $\begin{array}{c}\text { No. of } \\
\text { cases }\end{array}$ & $\begin{array}{c}\text { Micropapillary pattern } \\
\text { status }\end{array}$ \\
\hline $0 \%$ & 132 & Negative group $(n=164)$ \\
$<1 \%$ & 32 & Positive group $(n=21)$ \\
$\geq 1-<5 \%$ & 15 & \\
$\geq 5 \%$ & 6 & \\
\hline
\end{tabular}

micropapillary pattern in the specimen of largest area (Table 2). Cases with a micropapillary pattern ratio of more than $1 \%$ were categorized as micropapillary pattern positive (21 cases) and the remaining cases were considered to be micropapillary pattern negative (164 cases). In the micropapillary patternpositive cases, a micropapillary pattern was detected in space entrapped by atypical cells in seven cases (inside pattern: Figure 1d) and in the normal alveolar space in 11 cases (outside pattern: Figure 1e). A mixed pattern was detected in three cases.

\section{Relationship Between Clinicopathological Factors and Micropapillary Pattern}

TNM stage, $P$-factor, tumor size, lymph node metastasis, lymphatic invasion, venous invasion and serum CEA level at the preoperative stage all showed a correlation with disease-free interval and overall survival, and sex correlated with disease-free interval. TNM stage $(P=0.0002)$, lymphatic invasion $(P=0.0002)$ and lymph node metastasis $(P=0.03)$ were correlated with a micropapillary pattern-positive status (Table 1).

\section{Correlation Between Micropapillary Pattern Status and Outcome}

Of the 21 micropapillary pattern-positive cases, recurrence was observed in 13 patients and eight patients died during the follow-up period. The median and mean length of survival for all patients with small-size lung adenocarcinoma who underwent surgery were 62 and 62.6 months, respectively. Disease-free curves for micropapillary pattern-positive and micropapillary pattern-negative patients are shown in Figure 2a; the disease-free curve for the micropapillary pattern-positive group was significantly lower than that for the micropapillary pattern-negative group $(P=0.003)$. Overall survival curves for micropapillary pattern-positive and micropapillary pattern-negative patients are shown in Figure 2b; these curves indicate that the micropapillary pattern-positive group had significantly poorer survival than the micropapillary patternnegative group $(P=0.047)$. A comparison of micropapillary pattern-positive and micropapillary pattern-negative patients was also performed for stage a
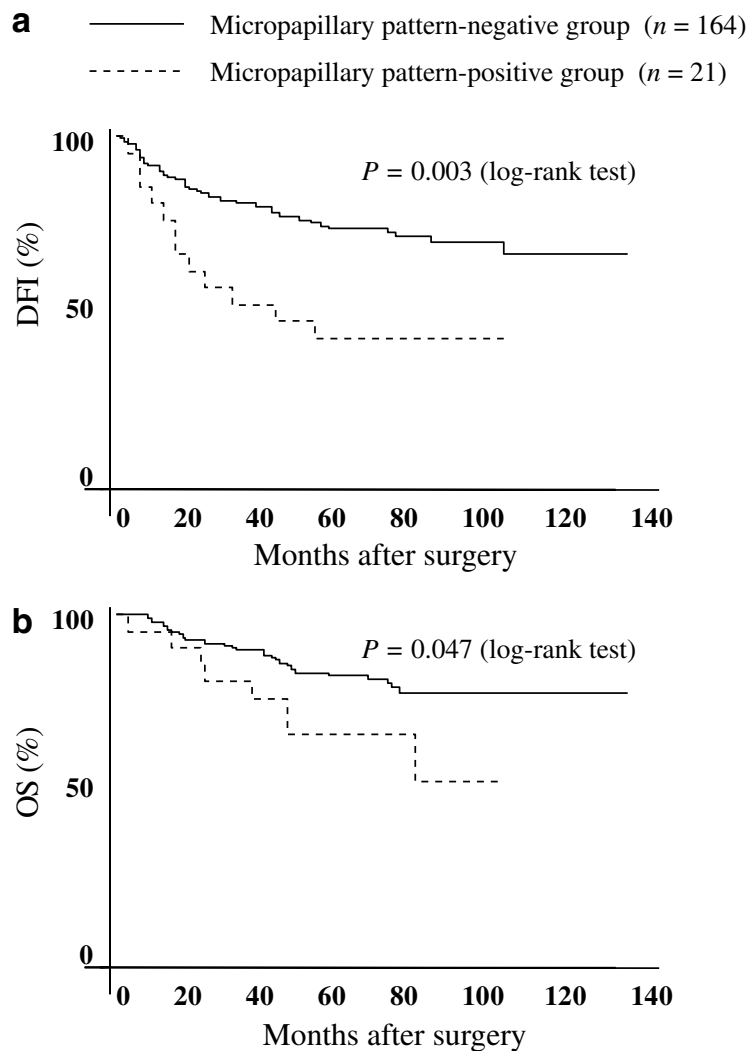

Figure 2 Disease-free interval and survival curves for micropapillary pattern-positive (micropapillary pattern ratio $\geq 1 \%$ ) and micropapillary pattern-negative (micropapillary pattern ratio $<1 \%$ ) cases. Kaplan-Meier plots are shown for postoperative disease-free interval (a) and survival (b). Log-rank analysis indicated significant differences in disease-free interval $(P=0.0002)$ and survival $(P<0.05)$ between the two groups.

IA cases only, in which disease-free interval and survival were longer than for other stages (Table 1). The disease-free curve for micropapillary patternpositive stage IA cases $(n=7)$ was significantly lower than that for micropapillary pattern-negative stage IA cases $(n=124)(P<0.0001$, data not shown), whereas overall survival showed no significant difference between the two groups $(P=0.34)$.

\section{Outcome Based on Histological Type of Micropapillary Pattern}

Disease-free interval and overall survival did not differ significantly among cases with an inside-type, outside-type and mixed-type micropapillary pattern (Figure 3a and b), although patients with an outsidetype pattern tended to have a shorter disease-free interval and shorter overall survival.

MUC1, MUC4, MUC5AC, Surfactant Apoprotein A, ERBb2, CEA, p16 and p27 Expression in Small-Size Lung Adenocarcinoma

The results of immunohistochemical analysis of protein expression were initially obtained as a 

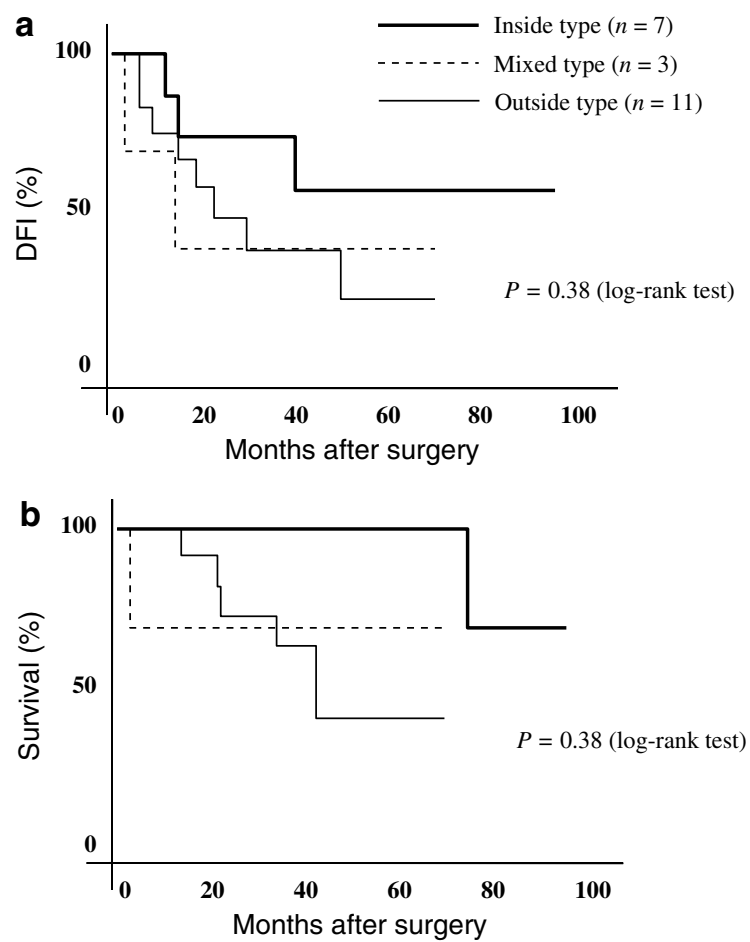

Figure 3 Disease-free interval and survival based on histological type of micropapillary pattern. Kaplan-Meier plots are shown for postoperative disease-free interval and survival for patients with micropapillary patterns of the inside, outside and mixed types. Log-rank analysis indicated no significant differences in diseasefree interval and survival among the three groups.

semiquantitative score (from 0 to +4 ). In Table 2, these data are shown in terms of high or low expression and in this form the data showed no significant correlation with micropapillary pattern status. Comparison of the semiquantitative expression score between micropapillary pattern and nonmicropapillary pattern areas suggested that MUC1 was expressed strongly in both areas in all micropapillary pattern-positive cases (Figure 4), although MUC4 expression in the micropapillary pattern area was lower than that in the non-micropapillary pattern area in five of the 21 cases. The expression levels of other proteins showed no significant difference between micropapillary pattern and non-micropapillary pattern areas. MUC1 was strongly expressed in the membrane of carcinoma cells in micropapillary patterns but not expressed in the cytoplasm.

\section{Surfactant Apoprotein a Expression and Outcome}

The combined status of MUC1 and surfactant apoprotein A expression was found to be an independent prognostic factor in our previous study. ${ }^{9}$ Therefore, we investigated MUC1 and surfactant apoprotein A expression in the micropapillary pattern area and performed statistical analysis using the Kaplan-Meier method for the 21 micro- papillary pattern-positive cases. Typical immunohistochemical findings are shown in Figure 5. As demonstrated in Figure $6 a$ and $b$, disease-free interval in patients with high surfactant apoprotein A expression (surfactant apoprotein $\mathrm{A}=4+$ ) was significantly longer than that in patients with low surfactant apoprotein A expression (surfactant apoprotein $\mathrm{A} \leq 3+)(P=0.03)$. However, survival in patients with high and low surfactant apoprotein A expression was not significantly different, and there was no case of recurrence or death in patients with high surfactant apoprotein A expression.

\section{Discussion}

The presence of a micropapillary pattern is known to lead to a poorer prognosis in cancer of the ovaries, ${ }^{10,11}$ breast $^{12,13}$ and bladder. ${ }^{14,15}$ Several studies have investigated the role of micropapillary patterns in lung adenocarcinoma, ${ }^{1-6}$ and here we have demonstrated that such patterns have unique characteristics in terms of MUC1 and surfactant apoprotein A expression. The criteria for definition of the micropapillary pattern ratio have been inconsistent in previous studies, ${ }^{1-6}$ with different methods used to evaluate this ratio. We determined the ratio in a more objective manner using NIH image software, and our criterion of a micropapillary pattern ratio $\geq 1 \%$ resulted in a lower percentage of micropapillary pattern-positive cases compared with previous reports. In addition, we excluded CD68-positive alveolar macrophages, which are sometimes difficult to distinguish from scattering cancer cells in alveolar space. Therefore, we believe results are representative of the percentage of micropapillary pattern-positive cases among a population of small-size lung adenocarcinoma patients.

Our results show that a micropapillary patternpositive status correlated with lymph vessel invasion and lymph node metastasis, leading to poor survival. In a previous study, we demonstrated that MUC1 downregulation by RNAi led to decreased proliferation and lymphatic metastasis, with an altered phenotype of pancreatic cancer cells. ${ }^{16}$ Since high MUC1 expression was detected on the entire surface of the micropapillary pattern in the current study, collectively our results suggest that the presence of a micropapillary pattern may be correlated with lymph node metastasis due to high MUC1 expression. This may occur because mucins (the gene product of MUC1) contribute to tumor invasion by simultaneously disrupting existing interactions between opposing cells (anti-adhesion) and establishing new ligands for interaction between the invading cell and the adjoining cells (adhesion). Cell-surface-associated mucins are bound to cells by an integral transmembrane domain and have relatively short cytoplasmic tails that associate with cytoskeletal elements and cytosolic adaptor 


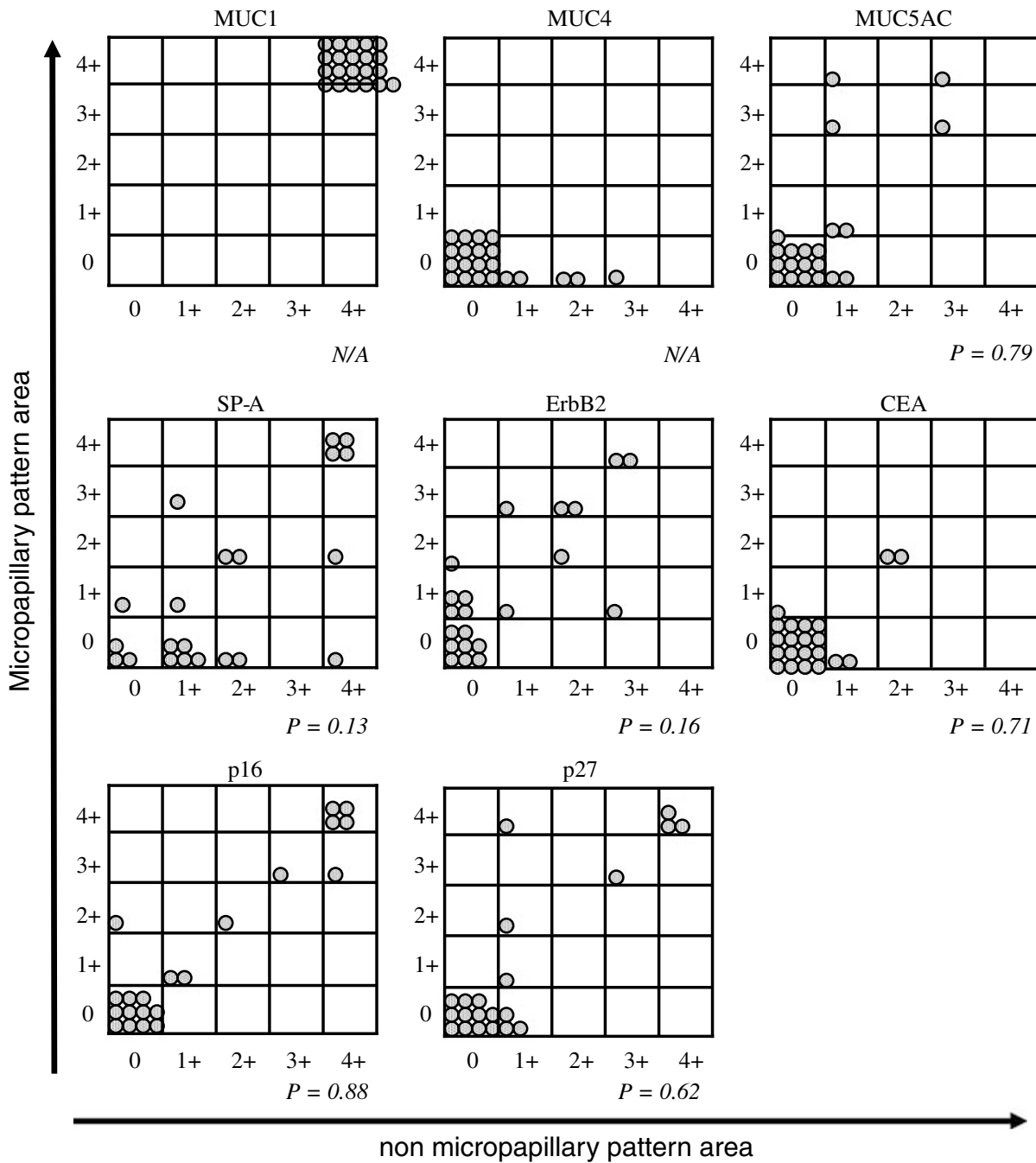

Figure 4 Expression status of MUC1, surfactant apoprotein A, MUC4, ERbB2, p27, CEA, MUC5AC and p16 in micropapillary pattern and non-micropapillary pattern areas in cases of primary small-size lung adenocarcinoma. The matrix shows the correlation of expression of each protein in the micropapillary pattern and non-micropapillary pattern areas in carcinoma tissue, based on immunohistochemical staining. The expression level was determined by the percentage of tumor cells that stained positively. MUC4 expression in micropapillary pattern areas was lower than in non-micropapillary pattern areas (Fisher's exact probability test, $P<0.05$ ). All other proteins showed no significant difference in expression between micropapillary pattern and non-micropapillary pattern areas. MUC1 showed strong expression in both areas.

proteins, and participate in signal transduction. Mucins might also serve as cell-surface receptors and sensors and conduct signals in response to external stimuli that lead to coordinated cellular responses that include proliferation, differentiation, apoptosis or secretion of specialized cellular products. ${ }^{17}$ Therefore, high MUC1 expression associated with a micropapillary pattern may make cancer cells more metastatically aggressive, even though the micropapillary pattern contains only a small number of cells.

We have previously reported that the prognosis of patients with small-size lung adenocarcinoma is poor for those with an expression pattern of MUC1 > surfactant apoprotein A, and conversely is good for an expression pattern of MUC1 $\leq$ surfactant apoprotein A. ${ }^{9}$ Jiang et al. ${ }^{18}$ reported that a deletion in the surfactant apoprotein A gene contributed to a poor prognosis in stage IA lung cancer; in the current study, the micropapillary pattern area always showed high MUC1 expression and loss of surfactant apoprotein A was predictive of a poor prognosis in cases with a micropapillary pattern.

We also compared the outcomes of cases with each type of micropapillary pattern: the inside and outside types. Cases with the outside type tended to have a poor prognosis, but there was no significance difference because of the low sample size The outside-type micropapillary pattern is present at the head of the growing tumor, and this may have a stronger effect on lymphatic invasion and lymph node metastasis compared with the inside type. Kuroda et al. ${ }^{19}$ described two types of micropapillary pattern in breast cancers based on breast-type 

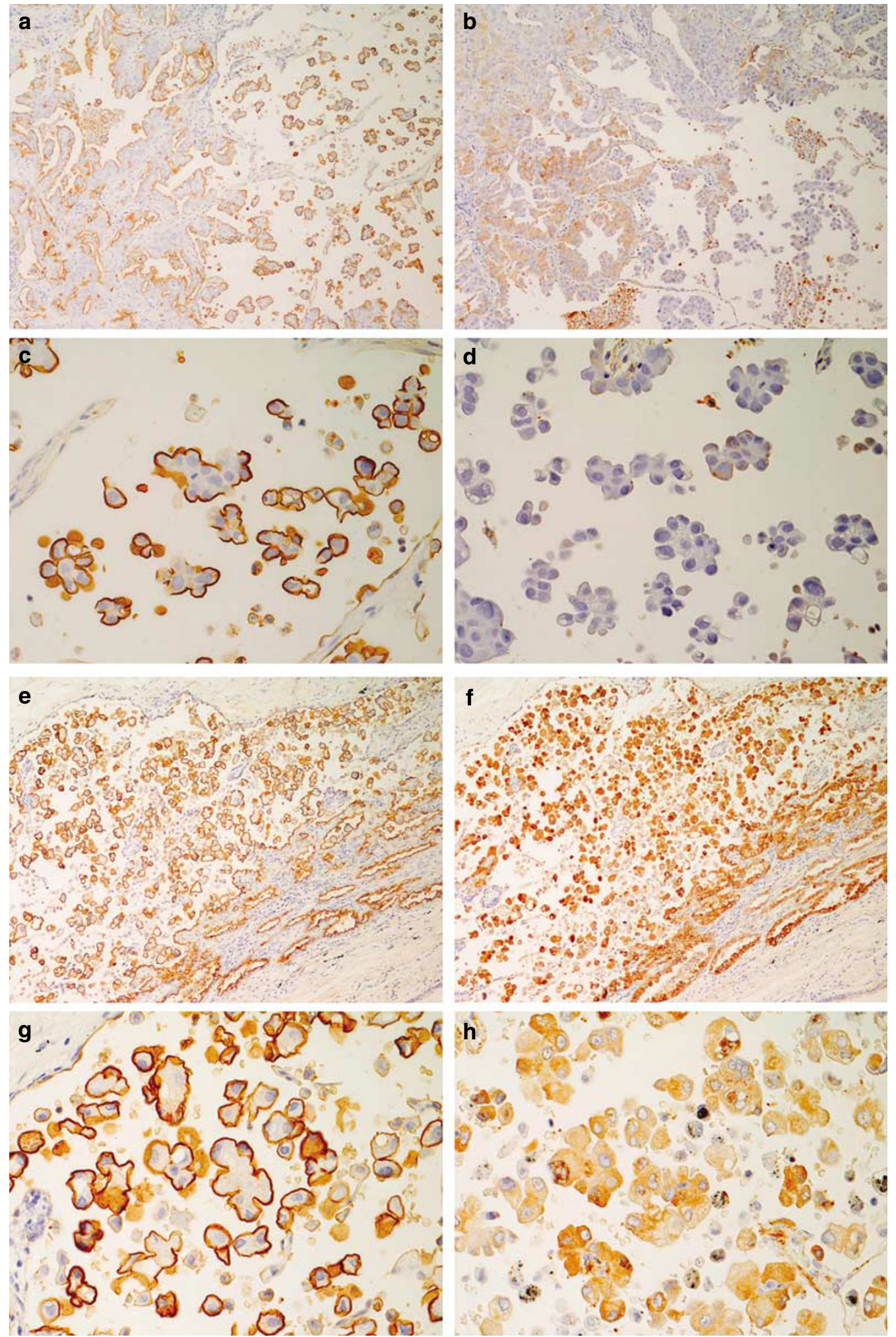

Figure 5 Expression of MUC1 and surfactant apoprotein A in carcinoma cells with micropapillary patterns (a, c, e, g: MUC1 immunohistochemistry; b, d, f, h: surfactant apoprotein A immunohistochemistry). $\mathbf{c}, \mathbf{d}, \mathbf{g}, \mathbf{h}$ are high-magnification images of $\mathbf{a}, \mathbf{b}, \mathbf{e}, \mathbf{f}$, respectively. MUC1 showed high expression over the whole surface of the micropapillary pattern (a, $\mathbf{c}, \mathbf{e}, \mathbf{g})$, while surfactant apoprotein A expression tended to be low in micropapillary pattern areas $(\mathbf{b}, \mathbf{d})$, with some exceptions $(\mathbf{f}, \mathbf{h})$. Surfactant apoprotein A was expressed mainly in the cytoplasm of cancer cells in the micropapillary pattern area (f, $\mathbf{h})$. 


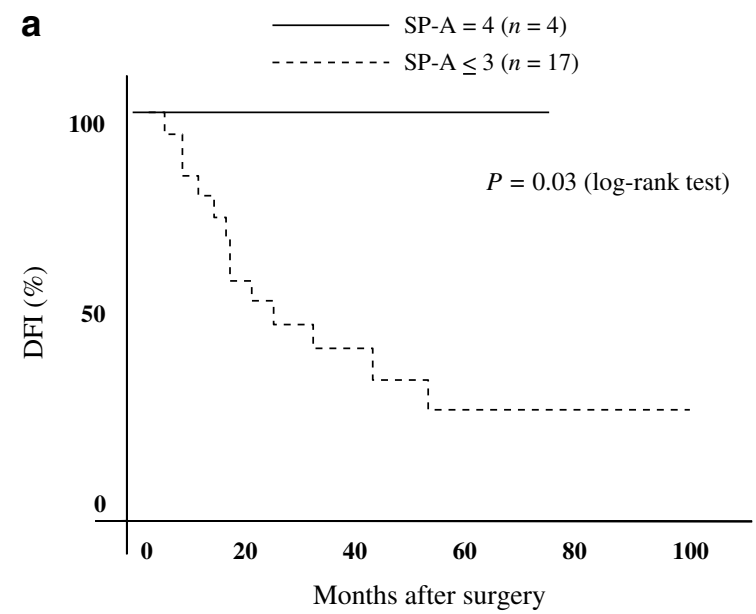

Table 3 Correlation between micropapillary pattern status and gene expression levels in small-size lung adenocarcinoma

\begin{tabular}{|c|c|c|c|c|c|}
\hline \multicolumn{2}{|c|}{ Gene expression } & \multirow[t]{2}{*}{$\begin{array}{c}\text { No. of } \\
\text { patients }\end{array}$} & \multicolumn{2}{|c|}{$\begin{array}{l}\text { Micropapillary } \\
\text { pattern status }\end{array}$} & \multirow[t]{2}{*}{ P-value } \\
\hline & & & $\begin{array}{l}\text { Negative } \\
(\mathrm{n}=164)\end{array}$ & $\begin{array}{l}\text { Positive } \\
(\mathrm{n}=21)\end{array}$ & \\
\hline \multirow[t]{2}{*}{ MUC1 } & Low $(0-3+)$ & 48 & $14(7.6)$ & $0(0)$ & \multirow[t]{2}{*}{0.16} \\
\hline & High $(4+)$ & 137 & $150(81.1)$ & $21(11.4)$ & \\
\hline \multicolumn{6}{|c|}{ Surfactant apoprotein } \\
\hline \multirow[t]{2}{*}{ A } & Low $(0-2+)$ & 132 & $117(63.2)$ & $15(8.1)$ & \multirow[t]{2}{*}{0.99} \\
\hline & High $(3+, 4+)$ & 53 & $47(25.4)$ & $6(3.2)$ & \\
\hline \multirow[t]{2}{*}{ MUC4 } & Low $(0,1+)$ & 160 & $142(76.8)$ & $18(9.7)$ & \multirow[t]{2}{*}{0.91} \\
\hline & High $(2+-4+)$ & 25 & $22(11.9)$ & $3(1.6)$ & \\
\hline \multirow[t]{2}{*}{ ERBb2 } & Low $(0,1+)$ & 132 & $117(67.6)$ & $15(8.7)$ & \multirow[t]{2}{*}{0.88} \\
\hline & High $(2+-4+)$ & 41 & $36(20.8)$ & $5(2.9)$ & \\
\hline \multirow[t]{2}{*}{ p27 } & Low $(0,1+)$ & 115 & $98(56.7)$ & $17(9.8)$ & \multirow[t]{2}{*}{0.06} \\
\hline & $\operatorname{High}(2+-4+)$ & 58 & $55(31.8)$ & $3(1.7)$ & \\
\hline \multirow[t]{2}{*}{ CEA } & Low (0) & 153 & $136(73.9)$ & $17(9.2)$ & \multirow[t]{2}{*}{0.77} \\
\hline & High $(1+-4+)$ & 31 & $27(14.7)$ & $4(2.2)$ & \\
\hline \multirow[t]{2}{*}{ p16 } & Low $(0,1+)$ & 85 & $72(40.0)$ & $13(7.2)$ & \multirow[t]{2}{*}{0.09} \\
\hline & High $(2+-4+)$ & 95 & 88 (48.9) & $7(3.9)$ & \\
\hline \multirow[t]{2}{*}{ MUC5AC } & Low $(0-2+)$ & 150 & $131(70.8)$ & $19(10.3)$ & \multirow[t]{2}{*}{0.24} \\
\hline & High $(3+, 4+)$ & 35 & $33(17.8)$ & $2(1.1)$ & \\
\hline
\end{tabular}

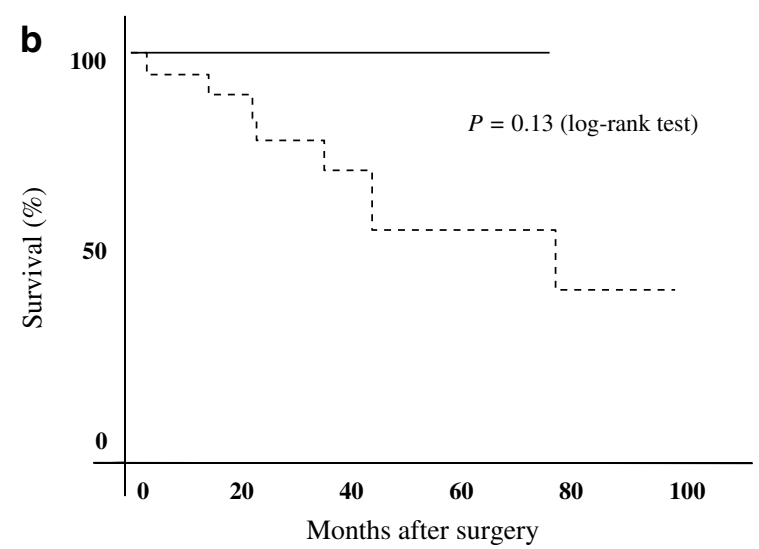

Figure 6 Disease-free interval and survival in relation to surfactant apoprotein A expression in small-size lung adenocarcinoma. Kaplan-Meier plots are shown for postoperative diseasefree interval (a) and survival (b) in cases with high surfactant apoprotein A expression (surfactant apoprotein $\mathrm{A}=4$ ) and lower surfactant apoprotein A expression (surfactant apoprotein $\mathrm{A} \leq 3$ ) in the micropapillary pattern area. Log-rank analysis indicated a significant difference in disease-free interval $(P=0.03)$ between these groups, but there were no cases of death in the high surfactant apoprotein A expression group.

and alveolar-type histology and showed that poorer prognosis was associated with a breast-type micropapillary pattern. If the inside type of micropapillary pattern corresponds to the breast type and the outside type corresponds to the alveolar type, our results are not in agreement with that of Kuroda and co-workers, making it important to clarify the definition of micropapillary pattern types. Furthermore, in breast cancer, it has been reported that MUC1 expression occurs in the whole cytoplasmic membrane in pseudo-invasive micropapillary carcinoma, whereas the reversed membrane pattern is present in pure invasive micropapillary carcinoma. ${ }^{20}$ In the current study, both the inside-type and outside-type micropapillary patterns showed a pure micropapillary pattern with MUC1 expression, again suggesting that further examination of the details of micropapillary patterns is required (Table 3).

It has been suggested that a micropapillary pattern can have a significant effect upon micrometastasis, ${ }^{6}$ and we found that the micropapillary pattern contributed to stage advancement through lymph node metastasis, leading to a poor prognosis. Furthermore, stage IA-positive cases had a shorter disease-free interval, but overall survival in stage IA was not affected by micropapillary pattern status. This result may be due to the relatively good overall prognosis in stage IA lung adenocarcinoma. Our findings are also compatible with those reported by Roh et al., ${ }^{6}$ who demonstrated that micropapillary pattern-positive cases show higher nodal micrometastasis in stage IA lung adenocarcinoma.

In conclusion, our immunohistological results indicate that strong expression of MUC1 across the surface of a micropapillary structure is a unique characteristic of a micropapillary pattern in lung adenocarcinoma. Recent reports ${ }^{1-6}$ have suggested that the presence of a micropapillary pattern may be an important prognostic factor in lung adenocarcinoma, and may be associated with lymphatic invasion and lymph node metastasis. Our results also show the significance of a micropapillary pattern in stage IA lung adenocarcinoma. Furthermore, reduced surfactant apoprotein A expression in the micropapillary pattern area contributed to a poor outcome in small-size lung adenocarcinoma, and the morphological pattern of the micropapillary pattern may play an important role in this disease. Finally, our results suggest various biological characteristics of a micropapillary pattern, which could be applicable in clinical practice. 


\section{Acknowledgements}

This study was supported in part by Grant-in-Aid 18014024 (S Yonezawa) from the Ministry of Education, Science, Sports, Culture and Technology, and the Kodama Memorial Fund for Medical Research, Japan. We are grateful to Mr Yoshiharu Atsuji, Ms Yoshiko Arimura and Ms Yukari Nishimura for their excellent technical assistance.

\section{Declaration}

Neither the submitted paper nor any similar paper, other than an abstract or preliminary communication, has been or will be submitted to or published in any other primary scientific journal. This manuscript has been read and approved by all of the authors. The requirements for authorship have been met, and each author believes that the manuscript represents honest work. We are aware that the cost of color reproduction is charged to the authors, and we approve these charges.

\section{References}

1 Amin MB, Tamboli P, Merchant SH, et al. Micropapillary component in lung adenocarcinoma: a distinctive histologic feature with possible prognostic significance. Am J Surg Pathol 2002;26:358-364.

2 Chantranuwat C. Metastatic potentiality of micropapillary and conventional histological patterns: a comparative study of 82 pulmonary adenocarcinomas. J Med Assoc Thai 2004;87(Suppl 2):S255-S260.

3 Hoshi R, Tsuzuku M, Horai T, et al. Micropapillary clusters in early-stage lung adenocarcinomas: a distinct cytologic sign of significantly poor prognosis. Cancer 2004;102:81-86.

4 Makimoto Y, Nabeshima K, Iwasaki H, et al. Micropapillary pattern: a distinct pathological marker to subclassify tumours with a significantly poor prognosis within small peripheral lung adenocarcinoma $(</=20 \mathrm{~mm})$ with mixed bronchioloalveolar and invasive subtypes (Noguchi's type C tumours). Histopathology 2005;46:677-684.

5 Miyoshi T, Satoh Y, Okumura S, et al. Early-stage lung adenocarcinomas with a micropapillary pattern, a distinct pathologic marker for a significantly poor prognosis. Am J Surg Pathol 2003;27:101-109.

6 Roh MS, Lee JI, Choi PJ, et al. Relationship between micropapillary component and micrometastasis in the regional lymph nodes of patients with stage I lung adenocarcinoma. Histopathology 2004;45:580-586.
7 Osako M, Yonezawa S, Siddiki B, et al. Immunohistochemical study of mucin carbohydrates and core proteins in human pancreatic tumors. Cancer 1993; 71:2191-2199.

8 Yamashita K, Yonezawa S, Tanaka S, et al. Immunohistochemical study of mucin carbohydrates and core proteins in hepatolithiasis and cholangiocarcinoma. Int J Cancer 1993;55:82-91.

9 Tsutsumida H, Goto M, Kitajima S, et al. Combined status of MUC1 mucin and surfactant apoprotein A expression can predict the outcome of patients with small-size lung adenocarcinoma. Histopathology 2004; 44:147-155.

10 Burks RT, Sherman ME, Kurman RJ. Micropapillary serous carcinoma of the ovary. A distinctive low-grade carcinoma related to serous borderline tumors. Am J Surg Pathol 1996;20:1319-1330.

11 Eichhorn JH, Bell DA, Young RH, et al. Ovarian serous borderline tumors with micropapillary and cribriform patterns: a study of 40 cases and comparison with 44 cases without these patterns. Am J Surg Pathol 1999; 23:397-409.

12 Middleton LP, Tressera F, Sobel ME, et al. Infiltrating micropapillary carcinoma of the breast. Mod Pathol 1999;12:499-504.

13 Walsh MM, Bleiweiss IJ. Invasive micropapillary carcinoma of the breast: eighty cases of an underrecognized entity. Hum Pathol 2001;32:583-589.

14 Amin MB, Ro JY, el-Sharkawy T, et al. Micropapillary variant of transitional cell carcinoma of the urinary bladder. Histologic pattern resembling ovarian papillary serous carcinoma. Am J Surg Pathol 1994;18: 1224-1232.

15 Johansson SL, Borghede G, Holmang S. Micropapillary bladder carcinoma: a clinicopathological study of 20 cases. J Urol 1999;161:1798-1802.

16 Tsutsumida H, Swanson BJ, Singh PK, et al. RNA interference suppression of MUC1 reduces the growth rate and metastatic phenotype of human pancreatic cancer cells. Clin Cancer Res 2006;12: 2976-2987.

17 Hollingsworth MA, Swanson BJ. Mucins in cancer: protection and control of the cell surface. Nat Rev Cancer 2004;4:45-60.

18 Jiang F, Caraway NP, Nebiyou Bekele B, et al. Surfactant protein A gene deletion and prognostics for patients with stage I non-small cell lung cancer. Clin Cancer Res 2005;11:5417-5424.

19 Kuroda N, Hamauzu T, Toi M, et al. Pulmonary adenocarcinoma with a micropapillary component: an immunohistochemical study. Case report. APMIS 2005;113:550-554.

20 Li YS, Kaneko M, Sakamoto DG, et al. The reversed apical pattern of MUC1 expression is characteristics of invasive micropapillary carcinoma of the breast. Breast Cancer 2006;13:58-63. 